\title{
The CUORICINO and CUORE double beta decay experiments
}

R. Ardito C. Arnaboldi , D.R. Artusa , F.T. Avignone III , M. Balata , I. Bandac , M. Barucci , J.W. Beeman , F. Bellini , C. Brofferio , C. Bucc , S. Capell ，F. Capozzi , L. Carbone , S. Cebrian , M. Clemenz , C. Cosmell , O. Cremonesi , R.J. Creswick , I. Dafine , A. de Waard , M. Diemo , M. Dolinsk , H.A. Farach , F. Ferroni , E. Fiorin ， S.J.F. Freedman ，G. Frossat , C. Gargiulo , A. Giuliani , P. Gorl , E. Guardincerri , T.D. Gutierrez , E.E. Haller ，K.M. Heege ， I.G. Irastorza , E. Longo , R. Maruyama , S. Morganti , S. Nis , C. None , E.B. Norman , A. Nucciotti , Nu Xu , E. Olivier , P. Ottonello , M. Pallavicin , E. Palmieri , M. Pavan , M. Pedretti , G. Pessina , S. Pirro , E. Previtali , L. Risegar , C. Rosenfeld , S. Sangiorgio , M. Sist , A.R. Smith , L. Torre ， G. Ventur ， L. Zanott 


\begin{abstract}
After an introduction on the various experimental techniques to be adopted in searches for double beta decay, the new approach based on the use of cryogenic low temperature detectors is described. The present results are reported on the limit for neutrinoless double beta decay of ${ }^{130} \mathrm{Te}$ obtained with the large bolometric detector CUORICINO. This setup consists of 44 cubic crystals of natural $\mathrm{TeO}_{2}$ with $5 \mathrm{~cm}$ sides and 18 crystals of $3 \times 3 \times 6 \mathrm{~cm}^{3}$. Four of these latter crystals are made with isotopically enriched materials: two in ${ }^{128} \mathrm{Te}$ and two others in ${ }^{130} \mathrm{Te}$. With a sensitive mass of $40.7 \mathrm{~kg}$, this array is by far the most massive running cryogenic detector. The array is operated at a temperature of $\sim 10 \mathrm{mK}$ in a dilution refrigerator under a heavy shield in the Gran Sasso Underground Laboratory at a depth of about 3500 m.w.e. The counting rate in the region of neutrinoless double beta decay is $0.18 \pm 0.02$ counts $\mathrm{keV}^{-1} \mathrm{~kg}^{-1} \mathrm{y}^{-1}$, among the lowest in this type of experiment. No evidence for neutrinoless double beta decay is found. The corresponding lower limit for the lifetime of this process is $2 \times 10^{24}$ years at $90 \%$ C.L. The resultant upper limit on the effective neutrino mass ranges between 0.2 and $1.0 \mathrm{eV}$, depending on the theoretically calculated nuclear matrix elements. This constraint is the most restrictive one, except for those obtained with Ge diodes, and is comparable to them. The second part of this report is devoted to the present status of the construction of the larger experiment CUORE (Cryogenic Underground Observatory for Rare Events) formed from 988 bolometers with a cubic $\mathrm{TeO}_{2}$ absorber of size $5 \times 5 \times 5 \mathrm{~cm}^{3}$, with a total mass of $\sim 750 \mathrm{~kg}$. We present technical details of the CUORE setup as well as of its location and our efforts to reduce radioactive backgrounds.
\end{abstract}

Keywords: Double beta decay; Neutrino mass

\title{
1. Introduction
}

The discovery of neutrino oscillations in solar, atmospheric, and reactor experiments [1-8] indicating that the mass of at least one neutrino is finite has strongly revived interest in neutrinoless double beta decay (DBD). This process in fact would not only indicate the nonconservation of the lepton number, but also provide a test to determine if neutrinos are majorana particles and, if so, enable us in this hypothesis to determine the absolute value of its mass. It becomes therefore imperative to search for a finite value for the effective electron neutrino mass [9-14]. In astrophysics, the recent results of the full sky microwave maps by WMAP, together with the $2 \mathrm{dF}$ Galaxy Redshift Survey [15], constrain the sum of the masses of the three neutrinos at $0.7-1.7 \mathrm{eV}$ [16-20]. A claim for a finite mass of $0.56 \mathrm{eV}$ has also been presented [21]. Direct experiments on single beta decay presently constrain the absolute value of this mass to less than $2.2 \mathrm{eV}$, while a bound of $\sim 0.2 \mathrm{eV}$ is expected in the KATRIN experiment [22-24].

A more restrictive limit for the effective mass of majorana neutrinos can undoubtly come from neutrinoless double beta decay (DBD). In its two negatron channel, DBD consists of the direct emission of two electrons from a nucleus $(\mathrm{A}, \mathrm{Z})$ decaying to the corresponding isobar $(A, Z+2)$. This process can be searched for when the single beta transition from $(A, Z)$ to $(A, Z+1)$ is energetically forbidden, or at least strongly suppressed, by a large change of the spinparity state. The process of two neutrino DBD is accompanied by the emission of two electron antineutrinos and therefore conserves lepton number. It is allowed by the standard model of electroweak interactions, and has been found in ten nuclei [24-30]. In contrast, conservation of the lepton number is violated in the majoron decay, where one or more massless Goldstone bosons accompany the emission of the two electrons, and in the so called neutrinoless DBD, 
where only the two electrons are emitted. In this case these two particles would share the total transition energy and a peak appears in the sum energy spectrum of the two electrons. In addition, the available phase space is much larger with respect to the two neutrino case, rendering neutrinoless DBD a very powerful way to search for lepton number non-conservation. The expected value for the effective neutrino mass, $\left\langle m_{v}\right\rangle$, or its upper limit, is proportional to the square root of the rate, which makes searches for neutrinoless DBD quite difficult. On the other hand, this rate is proportional to the square of the nuclear matrix element, whose evaluation is, at least at present, quite uncertain. Since the uncertainty in the value of the nuclear matrix element reflects itself directly in that of $\left\langle m_{v}\right\rangle$, searches for neutrinoless DBD should be carried out on several candidate nuclei. There is another important reason to do that. Natural and environmental radioactivity predict many peaks in any type of background spectrum. Even if a peak appears in the energy region predicted for neutrinoless DBD, one has to exclude the possibility that it could have been mimicked by one so far unknown radioactivity line. Only the presence of another peak at the different energy expected for DBD in other nuclei would unambiguously prove the existence of this very important process. No evidence has been claimed so far for the neutrinoless channel in any nucleus, with the exception of alleged evidence for neutrinoless DBD of ${ }^{76} \mathrm{Ge}$ reported by a subset of the Heidelberg-Moscow collaboration [31,32], but confronted by other authors [9,33,34], and even by a different subset of the same collaboration [35].The previous claim has however been enhanced by a more detailed analysis [36,37].

DBD can be searched for, indirectly, in radiochemical [38] or geochemical experiments [39-43], based on the search for the $(\mathrm{A}, \mathrm{Z}+2)$ product nuclei. These experiments are very sensitive, but indicate only the presence of the daughter nucleus and cannot therefore discriminate between lepton conserving and non-conserving processes or between decays to the ground or excited states of the daughter nucleus.

Direct experiments are based on two different approaches. In the source $\neq$ detector experiment, thin sheets of a double beta active material are inserted in a suitable detector. In the source $=$ detector or "calorimetric" experiments [44], the detector itself is made of a material containing the double beta active nucleus.

The use of cryogenic detectors to search for DBD was suggested in 1984 [45]. These bolometers, as shown in Fig. 1, are made [46-49] with diamagnetic and dielectric crystals. As a consequence, at low temperature their heat capacity is proportional to the cube of the ratio between the operating and Debye temperatures. In a cryogenic setup this capacity can become so small that even the tiny energy released by a particle in the form of heat generates a measurable temperature increase of the absorber. Cryogenic detectors offer a wide choice of DBD candidates, the only requirement being that the candidate nucleus is part of a compound which can be grown in the form of a crystal with the necessary thermal and mechanical properties. The isotope ${ }^{130} \mathrm{Te}$ is an excellent candidate to search for DBD due to its high transition energy $(2530.30 \pm 1.99 \mathrm{keV})$ [50] and large isotopic abundance (33.8\%) [51] which allows a sensitive experiment to be performed with natural tellurium. In addition, the expected signal at $2530.30 \mathrm{keV}$ happens to be in an energy region between the peak and the Compton edge of the ${ }^{208} \mathrm{Tl} \gamma$-rays at $2615 \mathrm{keV}$, which generally dominates the $\gamma$ background in this high energy region. Of the various compounds of this element, $\mathrm{TeO}_{2}$ appears to be the most promising due to good mechanical and thermal properties.

A series of experiments with various arrays of $340 \mathrm{~g}$ crystals of natural $\mathrm{TeO}_{2}$ have been performed in the Laboratori Nazionali del Gran Sasso. The results of an experiment carried out with an array of 16 crystals of natural Te and four enriched crystals, two in ${ }^{128} \mathrm{Te}$ and two in ${ }^{130} \mathrm{Te}$, with a total mass of $\sim 6.8 \mathrm{~kg}$ have been published [52]. 


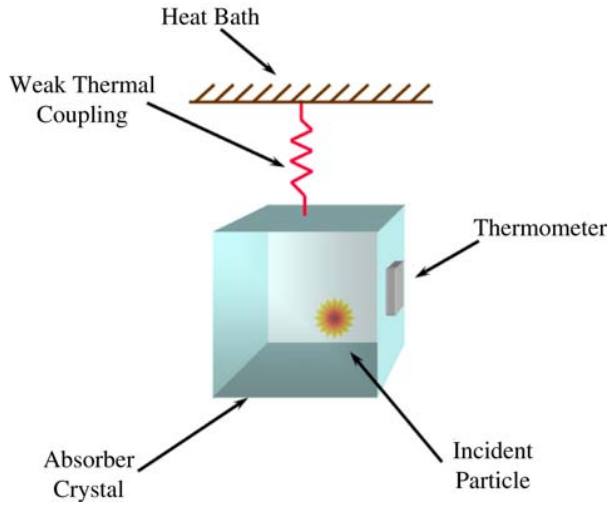

Fig. 1. Scheme of a thermal detector.
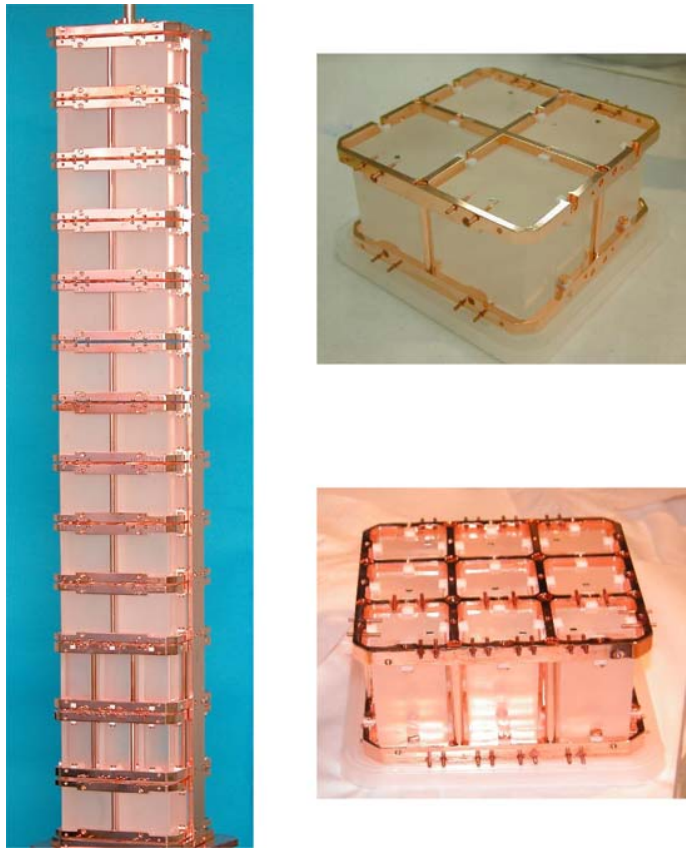

Fig. 2. The tower of CUORICINO and details of the planes with $5 \times 5 \times 5 \mathrm{~cm}^{3}$ and $3 \times 3 \times 6 \mathrm{~cm}^{3}$ bolometers.

\section{Experimental details}

The CUORICINO array consists of a tower with 13 planes containing 62 crystals of $\mathrm{TeO}_{2}$ operating in Hall A of the Gran Sasso Underground Laboratory [53] in the same dilution refrigerator previously used in our experiment with 20 crystals [52].

As shown in Fig. 2, the structure is as follows: the upper 10 planes and the lowest plane consist of four natural crystals of $5 \times 5 \times 5 \mathrm{~cm}^{3}$, while the 11 th and 12 th planes have nine $3 \times 3 \times 6 \mathrm{~cm}^{3}$ crystals each. In the $3 \times 3 \times 6 \mathrm{~cm}^{3}$ planes the central crystal is fully surrounded by the nearest neighbors. 


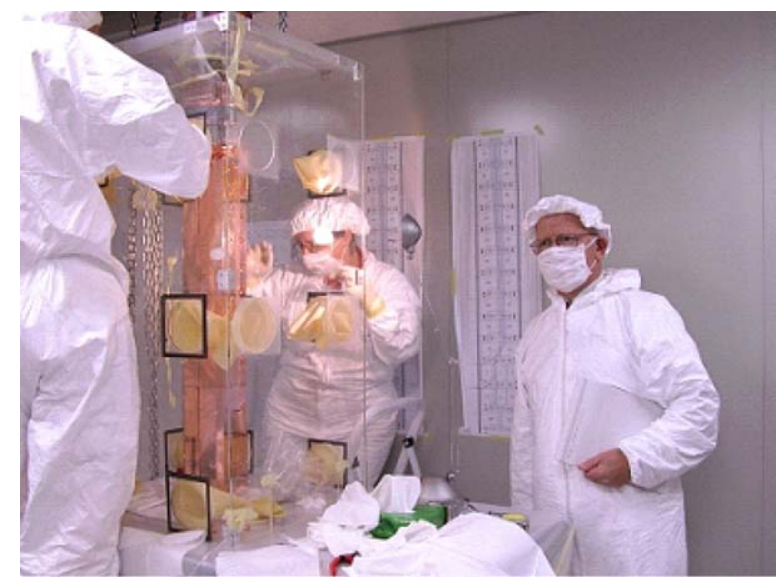

Fig. 3. Mounting of CUORICINO.

The small crystals are also made with natural tellurium, except for four of them. Two of these are enriched in ${ }^{128} \mathrm{Te}$ and two in ${ }^{130} \mathrm{Te}$, with isotopic abundances of $82.3 \%$ and $75 \%$, respectively. All crystals were grown with pre-tested low radioactivity material by the Shanghai Institute of Ceramics and shipped to Italy by sea in order to minimize the activation due to cosmic rays. They have then been lapped with specially selected low contamination abrasives to reduce the radioactive contamination on surfaces introduced by the original production process in China. All these operations and the final mounting of the tower were carried out in a nitrogen atmosphere glove box in a clean room, as shown in Fig. 3. The mechanical structure of the array was made exclusively with OFHC Copper and Teflon, also previously tested for measurable radioactive contaminations.

Thermal pulses are recorded by means of Neutron Transmutation Doped (NTD) Ge thermistors thermally coupled to each crystal and specifically prepared to present similar thermal performance. The gain of the bolometer is calibrated and stabilized by means of a resistor of $50-100 \mathrm{k} \Omega$, attached to each absorber and acting as a heater [54]. The tower is mechanically decoupled from the cryostat in order to avoid vibrations from the overall facility. This reduces noise in the detectors. It is therefore connected through a $25 \mathrm{~cm}$ copper bar to a steel spring fixed to the $50 \mathrm{mK}$ plate of the same dilution refrigerator previously used in the experiment with the 20 detector array [52]. The entire setup is shielded with two layers of lead of $10 \mathrm{~cm}$ minimum thickness each. The outer layer is made of common low-radioactivity lead, the inner of special lead with a contamination of $16 \pm 4 \mathrm{~Bq} / \mathrm{kg}$ in ${ }^{210} \mathrm{~Pb}$. The electrolytic copper of the refrigerator's thermal shields provides an additional shield of $2 \mathrm{~cm}$ minimum thickness. An external $10 \mathrm{~cm}$ layer of borated polyethylene has been installed to reduce the background due to environmental neutrons. In order to shield against the intrinsic radioactive contamination of the dilution unit's materials (e.g. from silver and stainless steel), an internal layer of $10 \mathrm{~cm}$ of Roman lead $\left({ }^{210} \mathrm{~Pb}\right.$ activity $<4 \mathrm{mBq} / \mathrm{kg}$ [55]) is located inside the cryostat immediately above the tower of the array. The lateral radioactivity due mainly to the thermal shields of the dilution refrigerator is reduced by a lateral internal shield of Roman lead of $1.2 \mathrm{~cm}$ minimum thickness. We should point out that, in order to use the same cryostat with the much larger CUORICINO array, the inner thermal shields had to be changed. As a consequence, the lateral layer of Roman lead had to be substantially reduced with respect to the configuration of the previous setup with 20 crystals. The corresponding worsening of the $2615 \mathrm{keV} \gamma$-ray background suppression was 
almost counterbalanced by the better quality of the new cryostat thermal shields. In fact, the rate of the 2615 line from ${ }^{208} \mathrm{Tl}$ has not substantially changed. We can therefore extrapolate that no additional contribution due to ${ }^{232} \mathrm{Th}$ contamination of the cryostat elements is expected in the neutrinoless DBD region. The refrigerator is surrounded by a Plexiglas anti-radon box flushed with clean $\mathrm{N}_{2}$ from a liquid nitrogen evaporator, and by a Faraday cage to eliminate electromagnetic interference.

The front-end electronics of all $3 \times 3 \times 6 \mathrm{~cm}^{3}$ detectors and of 20 of the 44 detectors of $5 \times 5 \times 5 \mathrm{~cm}^{3}$ are located at room temperature. They consist of a differential voltage sensitive preamplifier followed by a second stage and an anti-aliasing filter [54,56,57]. The differential configuration has been adopted to minimize signal cross talk and microphonic noise coming from the connecting wires. Precautions have been taken to suppress any possible effect coming from room temperature drift [54] and main power supply instability [58]. A pair of load resistors serves to bias each bolometer in a symmetric way [59]. All the necessary settings for the frontend and the biasing system are programmed remotely via computer, to allow the optimization of the overall dynamic performance separately for each detector [56]. The so-called cold electronics have been applied to 24 of the $5 \times 5 \times 5 \mathrm{~cm}^{3}$ detectors. In this case the preamplifier is located near the detector in a box kept at $\sim 100 \mathrm{~K}$ to reduce the wire lengths and therefore the noise due to microphonics, which is particularly dangerous in the low energy region of the spectrum, relevant to searches for interactions of weakly interacting massive particles (WIMPs).

The array was cooled down to temperatures of around $8 \mathrm{mK}$ with a temperature spread of $\sim 1 \mathrm{mK}$ among the different detectors. A routine calibration was performed using two wires of thoriated tungsten inserted inside the external lead shield in immediate contact with the Outside Vacuum Chamber of the dilution refrigerator. This calibration, which normally lasts one to two days, is performed at the beginning and end of each run, which usually lasts two weeks. In all the runs carried out so far, the average difference between the starting and ending calibrations was less than the experimental error in the peaks position evaluation (a fraction of a keV). An indirect check of the detector response's stability and reproducibility during the whole measurement period was also obtained from the analysis of the background sum spectrum. In fact, the positions of the environmental radioactivity $\gamma$ lines are in agreement with the corresponding ones observed in the calibration, without any sizeable worsening of the energy resolution.

\section{CUORICINO performance}

CUORICINO was cooled down at the beginning of 2003. Unfortunately, during this operation, electrical connections to 12 of the $445 \times 5 \times 5 \mathrm{~cm}^{3}$ detectors and to one of the $3 \times 3 \times 6 \mathrm{~cm}^{3}$ crystals were lost [60]. This was mainly due to an annoying problem with the soldering of the read-out wires at low temperature at the various stage of thermalization. New thermal links were therefore designed and tested. The cryostat was then warmed up in fall 2003 and all the thermalizers were changed. All but two channels were successfully recovered and CUORICINO was then cooled again in Spring 2004. The results presented here are from this first run [60] plus new data obtained in about 6 months [61]. The performance of the electrically connected detectors was found to be good: the average FWHM resolution during the calibration runs was $\sim 7 \mathrm{keV}$ and $\sim 9 \mathrm{keV}$, in the region of neutrinoless DBD for the $5 \times 5 \times 5 \mathrm{~cm}^{3}$ and the $3 \times 3 \times 6 \mathrm{~cm}^{3}$ crystals, respectively. The improved resolution of the new larger crystals with respect to that of the smaller ones is due to their better quality and thermal coupling. The summed calibration spectra of the large and smaller crystals are shown in Fig. 4. Double beta decay measurements started in April 2003 and were interrupted after three months due to the disconnection of the cooling water supply of 

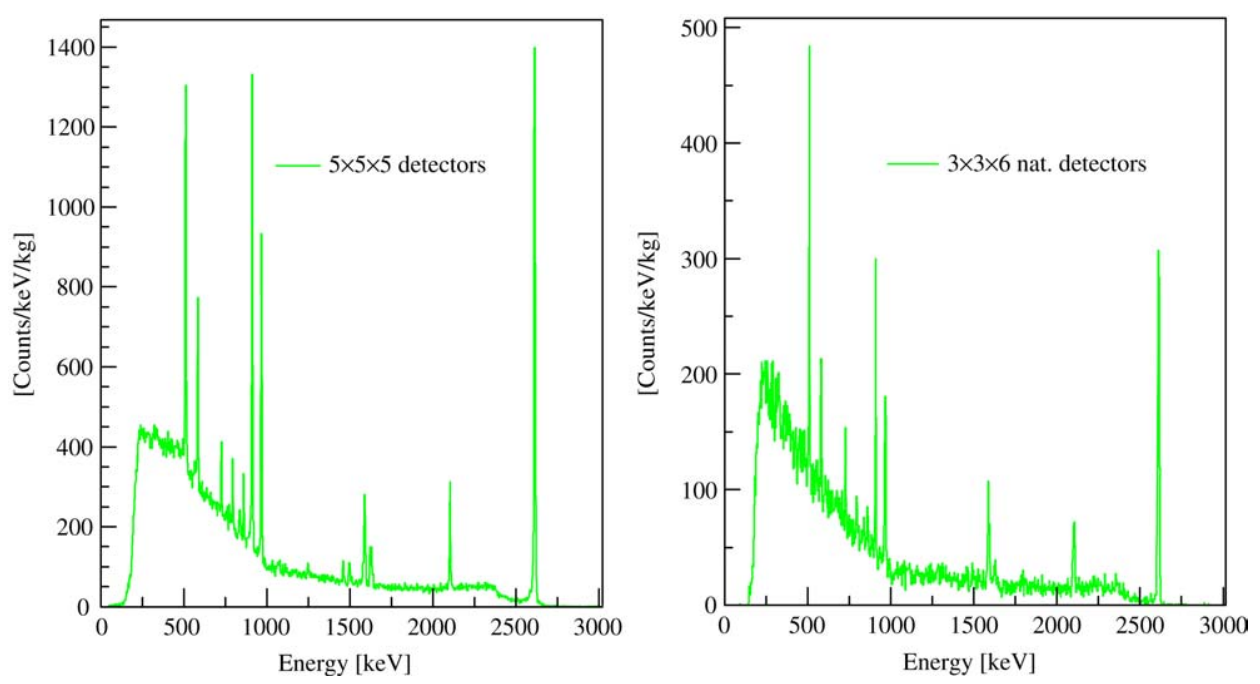

Fig. 4. Sum of the calibration spectra of the $5 \times 5 \times 5 \mathrm{~cm}^{3}$ and $3 \times 3 \times 6 \mathrm{~cm}^{3}$ crystals.

the laboratory. This was a result of environmental problems associated with the laboratory itself. During the last period, CUORICINO operated with a duty factor of $72 \%$, which is considered to be very good for a large cryogenic experiment [62].

\section{Results of CUORICINO}

The results reported here refer to the statistics collected up to July 31, 2005 corresponding to $\sim 5.87 \mathrm{~kg}$ y of ${ }^{130} \mathrm{Te}$. The calibration spectra for the ensemble of large and small detectors are shown in Fig. 4. We would like to point out that the enriched crystals were introduced in the CUORICINO setup to investigate the two neutrino DBD. Even if the present statistics are still inadequate to achieve that aim, it indicates a considerable improvement in the background in the low energy region with respect to that found in our previous experiment [52]. This first run of CUORICINO was optimized to investigate the high energy region relevant for neutrinoless DBD. However, we found that, even in the low energy region, which could be relevant for WIMPs searches, the background was reduced by about a factor of two with respect to the previous experiment [52]. This item will be investigated further in the future runs of CUORICINO.

The sum background spectrum of the $5 \times 5 \times 5 \mathrm{~cm}^{3}$ and $3 \times 3 \times 6 \mathrm{~cm}^{3}$ crystals in the region of neutrinoless DBD is shown Fig. 5. The background counting rates in the region of neutrinoless DBD are $0.18 \pm 0.02$ counts $\mathrm{keV}^{-1} \mathrm{~kg}^{-1} \mathrm{y}^{-1}$. These values are among the best ever obtained in this energy region and similar to those reached in experiments with Ge diodes [24-29]. No peak appears in the region of neutrinoless DBD of ${ }^{130} \mathrm{Te}$. A maximum likelihood procedure, used to establish the maximum number of $\beta \beta(0 v)$ events compatible to the measured background, implies an upper limit of $2.0 \times 10^{24}$ years for neutrinoless DBD of ${ }^{130} \mathrm{Te}$ at $90 \%$ C.L.

The limits that can be extracted from our result on the effective neutrino mass depend strongly on the results of the various theoretical calculations. We have considered all the most recent ones [61] apart from those based on the shell model, which have not been successful for heavy nuclei [63]. We have also considered the recent paper by Rodin et al. [64], whose calculations for neutrinoless DBD are based on the experimental values for the corresponding two neutrino channel. In fact, there is not yet a universally accepted value for the two neutrino DBD of 


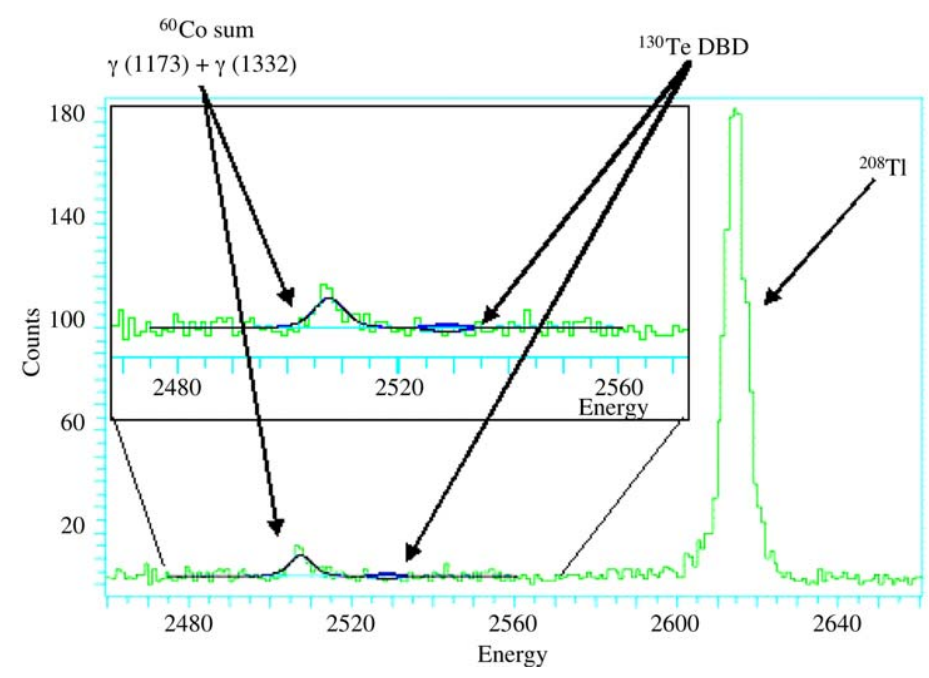

Fig. 5. Sum energy spectrum of the $5 \times 5 \times 5 \mathrm{~cm}^{3}$ and $3 \times 3 \times 6 \mathrm{~cm}^{3}$ crystals in the region of neutrinoless double beta decay.

${ }^{130} \mathrm{Te}$, and some concern on using the corresponding two neutrino channel has been published by Suhonen [65].

Taking into account theoretical uncertainties, we obtain from our data constraints in the ranges $(0.2-1) \mathrm{eV}$ at the 90 C.L. This range of values covers almost completely the range of bounds from 0.1 to $0.9 \mathrm{eV}$ corresponding to the evidence claimed by Klapdor-Kleingrothaus et al. [36,37]. Fig. 6 compares our result with the predictions from neutrino oscillations and with the evidence claimed by Klapdor-Kleingrothaus et al.

\section{Future experiments and prospects of CUORE}

A list of proposed second-generation experiments to search for neutrinoless DBD is reported in Table 1 . The predicted sensitivity to the neutrino mass is obviously strongly dependent on the value assumed for the nuclear matrix elements. From the experimental point of view, the predicted sensitivity depends, even more weakly, on the value assumed for the expected background, which is a difficult and sometime debatable issue indeed.

We are concerned with the experiment CUORE (Cryogenic Underground Experiment on Rare Events). The experimental facilities, including their location, have been approved by the Scientific Committee of the Gran Sasso Underground Laboratory, and are being designed and will be partially funded by the Italian Institute of Nuclear Physics. The scheme of CUORE [66-68] is shown in Fig. 7. It will be a packed array of 988 detectors with cubic absorbers of $\mathrm{TeO}_{2}$ of $5 \mathrm{~cm}$ each side. These will be arranged in 19 towers, very similar to CUORICINO, with 13 modules of four bolometers each. It will be shielded internally by a lateral layer of Roman lead of $33 \mathrm{~cm}$ thickness, plus a vertical disk, also of Roman lead, of $10 \mathrm{~cm}$ thickness.

Intense R\&D activity is going on with another dilution refrigerator operating in Hall $\mathrm{C}$ of the Gran Sasso Underground Laboratory and in the various groups of the Collaboration. The main issue is the study of the background and of the various ways to reduce it. In fact, in order to reach the desired sensitivity, the background of CUORE should be reduced to 0.01 counts $\mathrm{keV}^{-1} \mathrm{~kg}^{-1} \mathrm{y}^{-1}$ or, more optimistically, to 0.001 counts $\mathrm{keV}^{-1} \mathrm{~kg}^{-1} \mathrm{y}^{-1}$. Information 


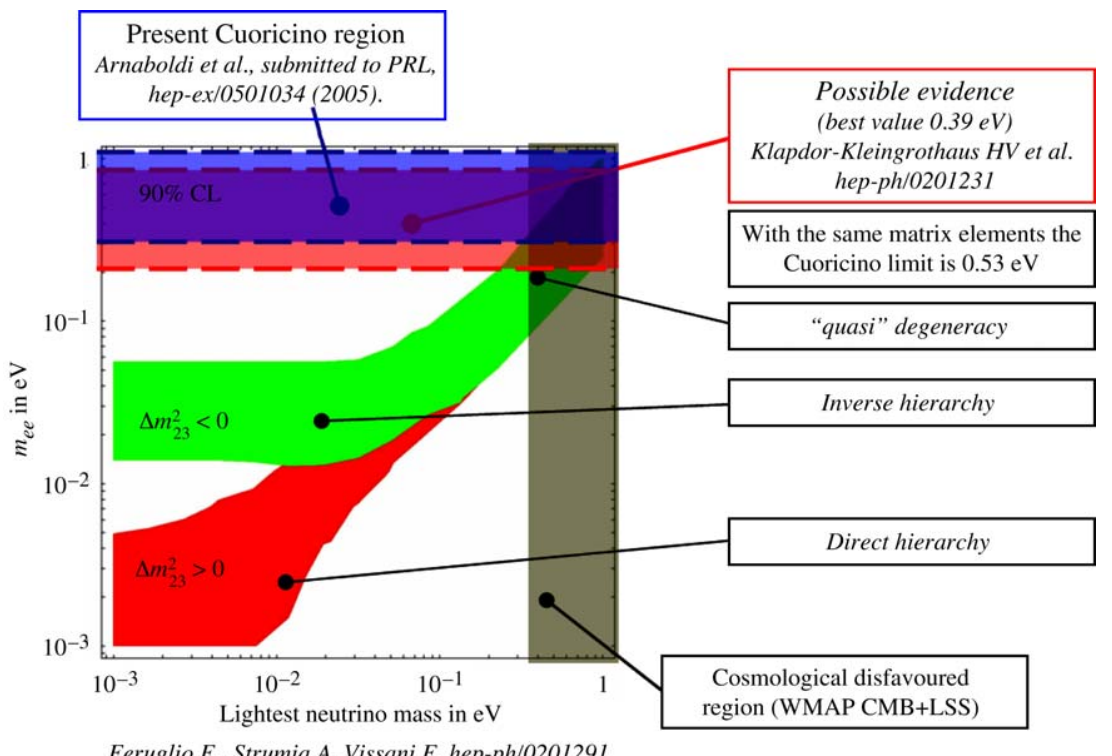

Fig. 6. Comparison between the CUORICINO results, the evidence of Klapdor-Kleingrothaus et al., and the indication from oscillations.

Table 1

Proposed future experiments on neutrinoless DBD and expected limits on $\left\langle m_{v}\right\rangle$

$\begin{array}{llllllr}\text { Experiment } & \text { Nucleus } & \% & \mathrm{Q} & \mathrm{T} & \text { Tech } & \left\langle m_{\nu}\right\rangle(\mathrm{meV}) \\ \text { CUORE } & { }^{130} \mathrm{Te} & 34 & 2529 & 9.2 \times 10^{25} & \text { Bolometric } & 9-57 \\ \text { GERDA } & { }^{76} \mathrm{Ge} & 7.8 & 2039 & 2 \times 10^{27} & \text { Ionization } & 29-94 \\ \text { Majorana } & { }^{76} \mathrm{Ge} & 7.8 & 2039 & 4 \times 10^{27} & \text { Ionization } & 21-67 \\ \text { GENIUS } & { }^{76} \mathrm{Ge} & 7.8 & 2039 & 1 \times 10^{28} & \text { Ionization } & 13-42 \\ \text { SuperNEMO } & { }^{8} \mathrm{Se} & 8.7 & 2995 & 2 \times 10^{26} & \text { Tracking } & 54-167 \\ \text { EXO } & { }^{136} \mathrm{Xe} & 8.9 & 2468 & 1.3 \times 10^{28} & \text { Tracking } & 12-31 \\ \text { MOON-3 } & { }^{100} \mathrm{Mo} & 9.6 & 3034 & 1.7 \times 10^{27} & \text { Tracking } & 13-48 \\ \text { DCBA } & { }^{150} \mathrm{Nd} & 5.6 & 3367 & 1 \times 10^{26} & \text { Tracking } & 16-22 \\ \text { Candles } & { }^{48} \mathrm{Ca} & .19 & 4272 & 3 \times 10^{27} & \text { Scintillation } & 29-54 \\ \text { CARVEL } & { }^{48} \mathrm{Ca} & .19 & 4272 & 1 \times 10^{27} & \text { Scintillation } & 50-94 \\ \text { GSO } & { }^{160} \mathrm{Gd} & 22 & 1730 & 1 \times 10^{26} & \text { Scintillation } & \sim 65 \\ \text { COBRA } & { }^{160} \mathrm{Gd} & 22 & 1730 & 1 \times 10^{26} & \text { Scintillation } & \text { Scintillation }\end{array}$

coming from the results of CUORICINO is vital from this point of view. As shown in Fig. 5, a considerable part of the background is above the well known $\gamma$ line of ${ }^{208} \mathrm{Tl}$ at $2615 \mathrm{keV}$ and is reasonably due to degraded $\alpha$ particles from surface contamination of the crystals and of the material in the immediate vicinity of it (mainly copper). A preliminary Monte Carlo calculation carried out on the CUORICINO results indicates that the main contributions to the background in the region of neutrinoless DBD comes from $\gamma$ radioactivity from the thermal shields of the dilution refrigerator surrounding the array $(\sim 30 \%)$, degraded $\alpha$ particles from the surface of the crystals $(\sim 10 \%)$, and the copper $(\sim 50 \%)$. The first of these contributions is due to the 


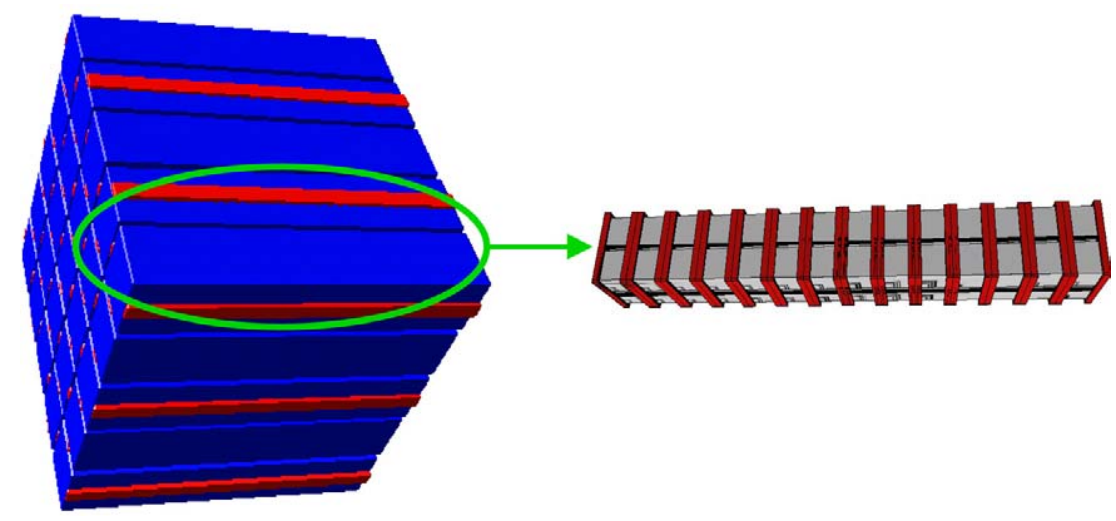

Fig. 7. Scheme of CUORE and of its installation.

already mentioned reduction of the Roman lead shield inside the refrigerator. In order to house CUORICINO in the dilution refrigerator used in a previous DBD experiment, we were forced to drastically reduce the layers of Roman lead inside the refrigerator between the CUORICINO array and the thermal shields which contained some radioactive contamination. This will not be the case for CUORE, where the $6 \mathrm{~cm}$ thick Roman lead shield will be amply sufficient to make this contribution negligible with respect to the others. A considerable presence of surface contamination of uranium and thorium on copper, extrapolated by Monte Carlo calculation from the CUORICINO spectrum, was confirmed by measurements with ICPMS and Laser Ablation ICPMS which indicate a surface contamination of around $1 \mathrm{ng} / \mathrm{g}$, at least three orders of magnitude larger than the bulk contamination of copper. A special run has been carried out in Hall $\mathrm{C}$ to investigate and attempt to decrease the surface contamination present in CUORICINO. The crystals were etched in ultrapure nitric acid and then lapped with ultrapure grinding powder, and copper was treated with ultrapure attacking and passivating liquids. With this procedure, we were able to decrease by a factor of four the contribution from the crystal surface, making it negligible with respect to the contribution from the outside material in contact with or facing the absorbers. In contrast, the latter was reduced by less than a factor of two. In order to ensure that this contribution would not come from materials different to copper but facing the crystals, we have performed a second run where four surfaces of the crystals were covered with Teflon. Two other faces were fully covered with heaters used for the stabilization of the gain and two others with the gold wires used for the electrical connections. The contribution of all these materials was found to be negligible, confirming copper as the main culprit. In a new, currently running measurement, we are covering all copper frames with ultrapure polyethylene to see if it could reduce the surface contribution from this metal. Other approaches are being considered, such as applying different cleaning procedure to copper, in particular plasma cleaning, which appears to be very promising. In addition to these passive methods to reduce the surface contamination, we are also considering two active methods.

The former (Fig. 8) consists of applying, in thermal contact with each absorber, bolometers formed by thin slabs (of germanium, silicon, or even $\mathrm{TeO}_{2}$ ) completely covering the absorber surfaces. If an event occurs in the bulk of an absorber, a normal pulse will be recorded both by the absorber and by the slab. If, on the contrary, an event involves the absorber surfaces, such as, for example, an $\alpha$ particle or a recoil from the surfaces of the crystals or of the copper frame, the risetime of the pulses from the slab bolometer will be much sharper that that from the absorber 


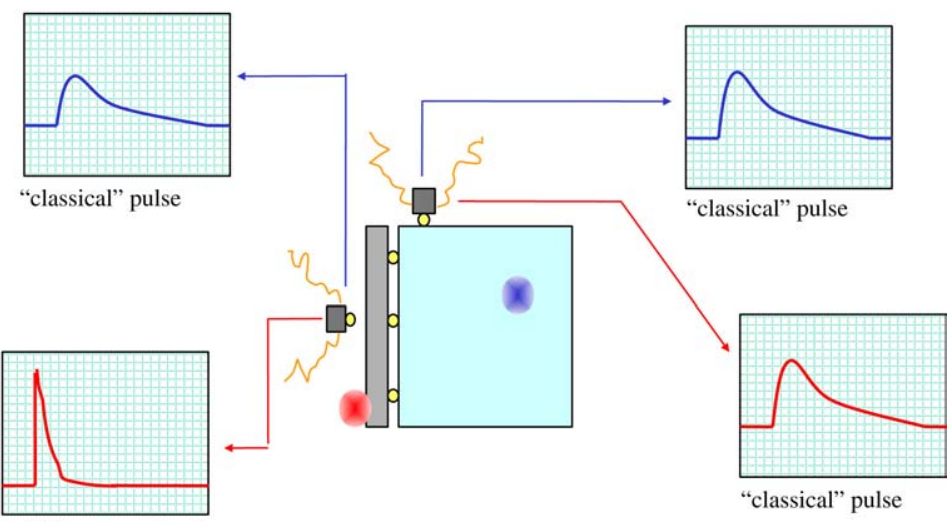

fast high saturated pulse

Energy deposited in the Ge crystal (degraded alpha event)

Fig. 8. Scheme of a Surface Sensitive Bolometer.

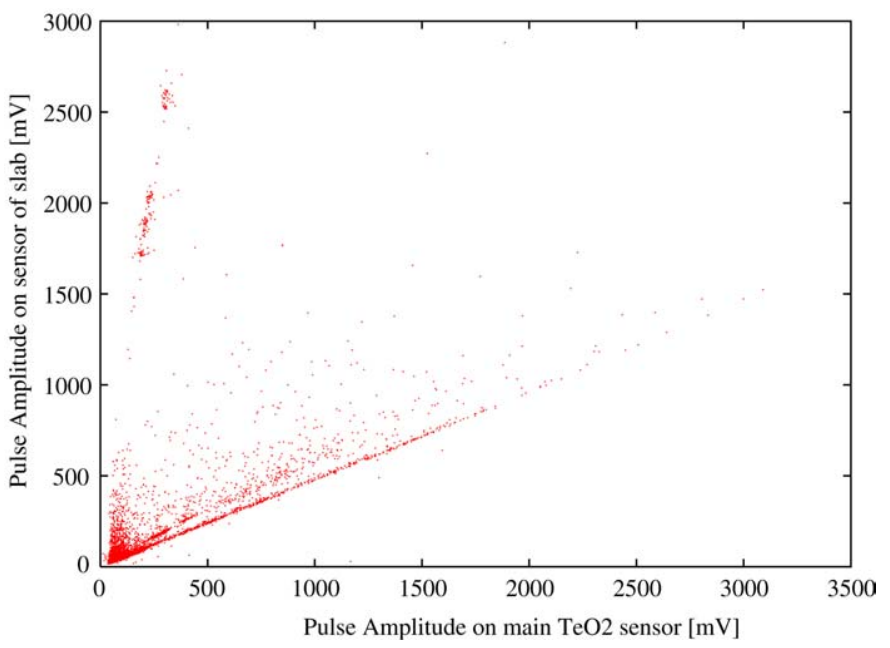

Fig. 9. Scatter plot from a Surface Sensitive Bolometer.

(Fig. 9). As shown in the scatter plot in Fig. 9, this would enable effective suppression of the surface contamination. We would like to add that a much simpler possibility would in principle exist, and could be exploited by simply thermally connecting the slab to the absorber with no thermistor on it. We have in fact found that the decay time of the absorber is different if the event occurs in the slab or in the bulk. This would enable pulse shape discrimination, even in a thermal detector.

The latter approach would consist in the use of the hybrid heat vs scintillation approach already successfully applied in experiments on Dark Matter such as CRESST or ROSEBUD, 
Table 2

Thermal detectors candidates for searches on DBD

Compound

${ }^{48} \mathrm{CaF}_{2}$

${ }^{72} \mathrm{Ge}$

${ }^{100} \mathrm{MoPbO}_{4}$

${ }^{116} \mathrm{CdWO}_{4}$

${ }^{130} \mathrm{TeO}_{2}$

${ }^{150} \mathrm{NdF}_{3}$

${ }^{150} \mathrm{NdGaO}_{3}$
Isotopic abundance (\%)

0.0187

7.44

9.63

7.49

34

5.64

5.64
Transition energy (keV)

4272

2038.7

3034

2804

2530

3368

3368

where nuclear recoils are selected by anticoincidence of the thermal (large) and scintillation (low) signals. In experiments on DBD, the approach is the opposite: to discard degraded $\alpha$ particles and recoils by imposing a coincidence between the heat pulse and the scintillation signal which is low for nuclear recoils and $\alpha$ particles, but large for ionizing events such as DBD. This approach has already been applied to $\mathrm{CaF}_{2}$ bolometer [69]. The group of Rome University (La Sapienza) [70] has successfully obtained considerable scintillation signals from crystals of $\mathrm{TeO}_{2}$ doped with $\mathrm{Nb}$ and $\mathrm{Mn}$.

\section{Present status and perspectives of CUORE}

CUORE was approved on April 2, 2004 by the Scientific Committee of the Gran Sasso Underground Laboratory. Its final location was decided on September of the same year. The almost contemporary approval by the advisory Commissione II of INFN (Italian Institute of Nuclear Physics) and allocation of funding allowed us to start R\&D activity in January 2005. A tender for the construction of the dilution refrigerator is underway. In August 2005, the Neutrino Scientific Assessment Group (NuSAG) in the United States stated that "The CUORE experiment has potential for good energy resolution and low background.... The schedule of CUORE is timely". The first priority for DBD experiments was given to CUORE, EXO and Majorana. Taking into account the uncertainty in the nuclear matrix elements, CUORE's sensitivity in $\left\langle m_{\nu}\right\rangle$ over a five year period is expected to range between $0.02 \mathrm{eV}$ and $0.10 \mathrm{eV}$ and between $0.011 \mathrm{eV}$ and $0.056 \mathrm{eV}$ under the hypothesis of a background of 0.01 counts $\mathrm{keV}^{-1} \mathrm{~kg}^{-1} \mathrm{y}^{-1}$ and 0.001 counts $\mathrm{keV}^{-1} \mathrm{~kg}^{-1} \mathrm{y}^{-1}$, respectively. These limits could be improved by $\sim 60 \%$ if the CUORE absorbers could be enriched in ${ }^{130} \mathrm{Te}$. We would like to add that, due to the flexibility of the thermal detectors, CUORE could also allow us to investigate neutrinoless DBD in other nuclei, even substituting one or more crystals of tellurite with absorbers containing other DBD candidates. The most promising compounds are reported in Table 2. All these compounds have been tested successfully with natural crystals as thermal detectors by the Milano group, with the exception of $\mathrm{NdF}_{3}$ and $\mathrm{NdGaO}_{3}$ which could not be brought to a sufficiently low temperature, probably due to the paramagnetic nature of neodymium.

\section{Conclusions}

Even if the evidence claimed by Klapdor-Kleingrothaus et al. is confirmed, we believe that the detection of at least another peak at a different transition energy expected for a different nuclear candidate is needed to definitely prove the existence of this lepton violating process. Thermal detectors are unique for this aim, in that they offer the possibility of measuring different 
DBD candidates. CUORICINO is the most sensitive experiment on neutrinoless DBD currently running. It has the same discovery potential as the Heidelberg-Moscow and IGEX experiments. However, to reach the sensitivity indicated by neutrino oscillation data under the inverse hierarchy hypothesis, a second-generation experiment is needed. The feasibility of CUORE has been proved by the excellent performance of CUORICINO, which represents substantially one of its towers. It can definitely reach this goal. The experiment has been approved and partially funded, has a definite underground location, and its installation there has already been designed. If funding is allocated in a reasonable time, data taking could start in 2010. The multidisciplinary aspects of thermal detectors allow one to investigate DBD candidates different to ${ }^{130} \mathrm{Te}$, as well as to search for other rare events such as interactions of WIMPs and solar axions and rare nuclear events.

\section{Acknowledgement}

Gorla and Torres were CEE fellows in the Network on Cryodetectors, under contract HPRN-CT-2002-00322. This experiment has been partly supported by the Commission of European Communities under contracts FMRX-CT98-03167 and HPRN-CT-2002-00322, by the U.S. Dept. of Energy under Contract number DE-AC03-76 SF000 98, and by the National Science Foundation.

\section{References}

[1] Q.R. Ahmad et al., Phys. Rev. Lett. 89 (2002) 001302 (and references therein); Q.R. Ahmad et al., arXiv:nucl-ex/0309004.

[2] S. Fukuda et al., Phys. Rev. Lett. 86 (2001) 5656 (and references therein); M.B. Smy et al., arXiv:hep-ex/0309011.

[3] K. Hagiwara et al., Phys. Rep. D 66 (2002) 1.

[4] K. Eguchi et al., Phys. Rev. Lett. 90 (2003) 021802.

[5] M.H. Ahn et al., Phys. Rev. Lett. 90 (2003) 041801 (and references therein).

[6] S.N. Ahmed et al., Phys. Rev. Lett. 92 (2004) 181301 (and references therein).

[7] H. Robertson, Solar neutrino experiments, lecture to this school.

[8] F. Suekane, The Kamland experiment, lecture to this school.

[9] F. Feruglio, A. Strumia, F. Vissani, Nuclear Phys. B 637 (2002) 345; Nucl. Phys. B 659 (2003) 359 (addendum) (also for reference therein).

[10] S. Pascoli, S.T. Petkov, Phys. Lett. B 544 (2004) 239; Phys. Lett. B 580 (2004) 280.

[11] R.R. Joaquim, Phys. Rev. D 68 (2003) 033019.

[12] C. Giunti, hep-ph,0308206, 2003.

[13] J. Bahcall et al., Phys. Rev. D 70 (2004) 033012.

[14] H. Murayama, C. Pena-Garay, Phys. Rev. D 69 (2004) 031301, and lecture by H. Murayama to this school.

[15] O. Elgaroy et al., Phys. Rev. Lett. 89 (2002) 061301.

[16] V. Barger et al., Phys. Lett. B 595 (2004) 55.

[17] S. Hannestad et al., J. Cosmol. Astrop. Phys. JCAP05 004 (2003).

[18] M. Tegmark et al., Phys. Rev. 69 (2004) 10350.

[19] D.N. Spergel et al., Astrophys. J. Suppl. 148 (2003) 175.

[20] P. Crotty et al., Phys. Rev. D 69 (2004) 123007.

[21] S.W. Allen et al., Mon. Not. R. Astron. Soc 346 (2003) 593.

[22] V.M. Lobashov, Nuclear Phys. A 719 (2003) 153.

[23] Ch. Weinheimer, Neutrino mass from tritium decay, lecture to this school.

[24] C. Aalseth et al., arXiv:hep-ph/0412300v1.

[25] S. Elliott, P. Vogel, Ann. Rev. Nucl. Part. Sci. 52 (2002) 115.

[26] O. Cremonesi, Nuclear Phys. B Proc. Suppl. 118 (2003) 287.

[27] V.I. Tretyak, Yu.G. Zdesenko, At. Data Nucl. Data Tables 80 (2002) 83.

[28] H. Ejiri, Prog. in Part. and Nuclear Phys. B 48 (2002) 185. 
[29] S. Elliott, J. Engel, J. Phys. G: Nucl. Part. Phys. 30 (2004) R183.

[30] F. Avignone, Evaluation of techniques for neutrinoless DBD experiments and prospect for direct observation, lecture to this school.

[31] H.V. Klapdor-Kleingrothaus, A. Dietz, H.L. Harney, I.V. Krivosheina, Modern Phys. Lett. A 16B (2001) 2409-2420;

H.V. Klapdor-Kleingrothaus, Reply to comment of article "Evidence for Neutrinoless Double Beta Decay", arXiv:hep-ph/0205228.

[32] L. Harney, Reply to the comment on "Evidence for neutrinoless double beta decay", arXiv:hep-ph/0205293.

[33] C.E. Aalseth et al., Modern Phys. A 17 (2002) 1475. hep-ex/0202018.

[34] Yu.G. Zdesenko, F.A. Danevic, V.I. Tretyak, Phys. Lett. 546 (2002) 206 (and references therein).

[35] A.M. Bakaliarov et al., Results of the experiment on investigation of Germanium-76 double beta decay, presented to the NANP2003, Dubna, June 24, 2003.

[36] H.V. Klapdor-Kleingrothaus et al., Phys. Lett. B 584 (2004) 189.

[37] H.V. Klapdor-Kleingrothaus et al., Nucl. Instrum. Methods 522 (2004) 367.

[38] A.L. Turkevich, T.E. Economou, G.A. Cowan, Phys. Rev. Lett. 67 (1992) 3211.

[39] O.K. Manuel, Proc. of the Int. Symp. on Nuclear Beta Decay and Neutrino, Osaka, Japan, World Scientific, Singapore, 1986, p. 71;

O.K. Manuel, J. Phys. G: Nuclear Part. Phys. 17 (1991) S221;

J.T. Lee, O.K. Manuel, R.I. Thorpe, Nucl. Phys. A 529 (1991) 29, and private communication by O.K. Manuel.

[40] T. Kirsten, Proc. of the Int. Symp. on Nuclear Beta Decay and Neutrino, Osaka, Japan, World Scientific, Singapore, 1986, p. 81, and private communication by T. Kirsten.

[41] T. Bernatowicz et al., Phys. Rev. Lett. 69 (1992) 2341; Phys. Rev. C 47 (1993) 806 (and references therein).

[42] N. Takaoka, Y. Motomamura, K. Nagao, Phys. Rev. C 53 (1996) 1557.

[43] A. Kawashima et al., Phys. Rev. C 47 (1993) 2452 (this indication has been confirmed in a direct experiment by the NEMO II collaboration [25]).

[44] G.F. Dell'Antonio, E. Fiorini, Suppl. Nuovo Cimento 17 (1960) 132.

[45] E. Fiorini, T. Niinikoski, Nucl. Instrum. Methods 224 (1984) 83.

[46] D. Twerenbold, Rep. Progr. Phys. 59 (1996) 349;

N. Booth, B. Cabrera, E. Fiorini, Ann. Rev. Nucl. Sci. 46 (1996) 471.

[47] F. Scott Porter, D. McCammon, M. Galeazzi, C.K. Stahle (Eds.), Proc. of the IX International Workshop on Low Temperature Detectors, Madison, Wisconsin, 22-27 July 2001, Amer. Inst. Phys. 605 (2002).

[48] F. Gatti (Ed.), Proc. of the X International Workshop on Low Temperature Detectors, Nucl. Instrum. Methods A 520 (2004).

[49] C. Enns (Ed.) Cryogenic Particler Detection, Springe Publishing Company (in press).

[50] G. Audi, A.H. Wapstra, C. Thibault, Nuclear Phys. A 729 (2003) 337.

[51] R.B. Firestone, C. Baglin, S.F. Chu, Tables of Isotopes, 8th edition, 1998 (1998 CD-ROM update).

[52] C. Arnaboldi et al., Phys. Lett. B 557 (2003) 167 (and references therein).

[53] A. Bettini, Phys. Uspekhi 170 (2001) 977 (and references therein).

[54] A. Alessandrello et al., IEEE Trans. Nuclear Science 44 (1997) 416.

[55] A. Alessandrello et al., Nucl. Instrum. Methods A 142 (1998) 454.

[56] A. Alessandrello et al., Nucl. Instrum. Methods 444 (2000) 111-114.

[57] G. Pessina, Nucl. Instrum. Methods 444 (2000) 132.

[58] G. Pessina, Rev. Sci. Instrum. 70 (1999) 3473.

[59] C. Arnaboldi et al., Low frequency noise characterization of very large value resistors, IEEE Trans. Nucl. Sci. (in press).

[60] C. Arnaboldi et al., Phys. Lett. B 584 (2004) 260.

[61] C. Arnaboldi et al., Phys. Rev. Lett. 95 (2005) 142501.

[62] C. Brofferio, Nucl. Instrum. Methods A 520 (2004) 151.

[63] A. Faessler, F. Simkovich, J. Phys. G: Nucl. Part. Phys. 24 (1998) 2139, and lecture by A. Faessler to this school.

[64] V.A. Rodin, A. Faessler, F. Simkovic, P. Vogel, nucl-th/0305005.

[65] J. Suhonen, Phys. Lett. B 607 (2005) 87.

[66] C. Arnaboldi et al., Astropart. Phys. 20 (2003) 91 (and references therein).

[67] C. Arnaboldi et al., Nucl. Instrum. Methods A 518 (2004).

[68] C. Arnaboldi et al., CUORE a Cryogenic Underground Observatory for rare Events, hep-ex/0501010, 2005 (and references therein).

[69] A. Alessandrello et al., Phys. Lett. B 420 (1999) 109.

[70] I. Dafinei et al., $\mathrm{TeO}_{2}$ scintillating crystal growth and properties, in: *th Int. Conf. on Inorganic Scintillators and their Industrial Applications SCINT2005; Crimea, Alushta (Ukraine0, September 19-23, 2005). 